\title{
Multiphoton absorption and anharmonicity
}

\author{
M. Persico \\ Dipartimento di Chimica, Università di Pisa, Via Risorgimento 35, I-56126 Pisa, Italy \\ P. Van Leuven \\ Departement Wiskunde-Informatica, Universiteit Antwerpen, Groenenborgerlaan 171, B-2200 Antwerpen, Belgium
}

(Received 19 June 1995)

\begin{abstract}
The multiphoton absorption mechanism of anharmonic molecular bonds in laser fields of high intensity is investigated. The time dependence of the absorbed energy as a function of field frequency is studied for a number of cases of varying anharmonicity and field strength. It is found that the short-time dynamics can be meaningfully described by an "apparent detuning." The results obtained with a continuous-wave field are used for the design of Gaussian pulses.

PACS number(s): $33.80 . \mathrm{Wz}$
\end{abstract}

\section{INTRODUCTION}

The interaction of atoms and molecules with short, intense laser pulses is presently a subject of study of considerable interest, not in the least because of the growing possibilities of time-resolved experiments using modern femtosecond techniques. The theoretical analysis of such experiments necessitates the solution of the time-dependent Schrödinger equation. To this end, during the past decade various analytical and numerical techniques have been developed and applied to realistic molecular systems. There is, however, also a need for simple generic nonperturbative models, which help in understanding the individual steps in the complicated mechanism of intramolecular dynamics.

Here we focus attention on the infrared multiphoton absorption of a diatomic molecule or an isolated molecular bond. It is well known that the anharmonicity of the vibration spectrum impedes the efficiency of this process because the field becomes more and more off resonance as one climbs through the anharmonically spaced higher levels. As real molecules are anharmonic, it is interesting to study systematically the effects of anharmonicity on the multiphoton absorption mechanism.

Previous investigations of the driven anharmonic oscillator (see [1]) have concentrated mostly on the correspondence between classical and quantum descriptions or on the influence of the field parameters at some fixed typical degree of anharmonicity. In this paper we wish to report on a number of numerical experiments, using a simple but realistic model problem, by which we study the time propagation of a wave packet in anharmonic potentials of different anharmonicity under the action of a time-dependent external field of high intensity.

It has been noticed before that the resonant multiphoton absorption is a long-time process. This means that if the molecule is exposed to the action of a resonant monochromatic field, the number of absorbed photons can still increase after a large number of recurrences of the quasiperiodic propagation of the wave packet. In view of the application of pulsed fields with short (femtosecond) duration, however, we focus in this paper on the short-time behavior of the system, which, depending on anharmonicity and field strength, is typically of the order of 20 optical cycles or a few hundred femtoseconds. We shall analyze the characteristics of the energy absorption mechanism as a function of anharmonicity, field strength, and field frequency using a square pulse and show that this analysis is useful to predict the behavior also under the action of more realistic pulse shapes such as Gaussian pulses.

In Sec. II we consider the problem of a molecular vibrator interacting with an external dipole field. We describe concisely a numerical algorithm to solve the time-dependent Schrödinger equation. We do not pretend that this is the most efficient computational method to tackle our problem. However, we have this one at our disposal [2] and we find it sufficiently accurate and rapid for our purpose.

In Sec. III we define a family of Morse potentials with varying anharmonicity. We give numerical results, which illustrate the role of anharmonicity in the multiphoton absorption process.

\section{MODEL AND METHOD}

Let us consider the simplified problem of a diatomic molecule with reduced mass $M$ and internuclear distance $R$ in an isolated electronic state and disregarding the influence of rotational motion. The relevant Schrödinger equation is then

$$
i \hbar \frac{\partial \psi(R, t)}{\partial t}=\left(H_{0}+H_{1}\right) \psi(R, t)
$$

where

$$
H_{0}=-\frac{\hbar^{2}}{2 M} \frac{\partial^{2}}{\partial R^{2}}+V(R)
$$

is the molecular Hamiltonian in the adiabatic approximation and $H_{1}$ is the interaction with the field. To simulate the anharmonicity we shall use the Morse potential with equilibrium distance $R_{0}$ and dissociation energy $D$

$$
V(R)=D\left\{1-\exp \left[-\alpha\left(R-R_{0}\right)\right]\right\}^{2} .
$$

It is customary to consider as a measure for the anharmonicity the so-called anharmonicity parameter 


$$
\beta=\frac{\hbar \omega}{4 D}
$$

where $\omega$ is the frequency of the harmonic oscillator corresponding to the quadratic approximation of $V$

$$
\omega=\alpha\left(\frac{2 D}{M}\right)^{1 / 2} .
$$

Although we could have simulated anharmonicity otherwise, e.g., by taking cubic or quartic oscillators, we have preferred the Morse potential because of its traditional use in molecular physics. With this choice we have linked anharmonicity to dissociation energy. In the context of our work here, however, dissociation is an unimportant phenomenon, quantitatively as well as qualitatively.

For the interaction with the external field we use the dipole approximation in the length gauge [3]

$$
H_{1}=-\mu(R) E(t)
$$

with dipole moment function $\mu(R)$ and electric field $E(t)$. In the one-dimensional model used here, the electric field is assumed to be linearly polarized along the internuclear axis. For the dipole moment function we shall use the linear approximation

$$
\mu(R)=q R,
$$

where $q$ is an effective charge.

The form (7) of the dipole function diverges for large $R$ and hence one might be suspicious about the validity of the linear approximation when very extended vibrational eigenfunctions enter into the calculation, i.e., for highly excited states in a strongly anharmonic potential. In order to monitor this effect we have also considered two ad hoc forms of the dipole function

$$
\begin{aligned}
& \mu_{1}(R)=q \frac{R^{2}}{R_{0}} \exp \left(-\frac{R-R_{0}}{R_{0}}\right), \\
& \mu_{2}(R)=q R_{0} \exp \left(-\frac{R-R_{0}}{R_{0}}\right) .
\end{aligned}
$$

They are chosen such that they converge to zero for large $R$ and have the properties

$$
\begin{gathered}
\mu_{1}\left(R_{0}\right)=q R_{0}=\mu_{2}\left(R_{0}\right), \\
\left(\frac{d \mu_{1}}{d R}\right)_{R_{0}}=q=-\left(\frac{d \mu_{2}}{d R}\right)_{R_{0}} .
\end{gathered}
$$

For the electric field we take a continuous wave with frequency $\Omega$

$$
E(t)=E \cos \Omega t
$$

It is assumed that this field is switched on and off suddenly at the initial and final times. In Sec. IV we shall also consider fields modulated by a Gaussian envelope. In order to test the insensitivity of the results to the phase of the field, we also considered a time dependence of the form $\sin \Omega t$ (phase shift of $\pi / 2$ ) while the difference between using $\mu_{1}$ and $\mu_{2}$ is, in a sense, equivalent to a phase shift of $\pi$.

As for the amplitude $E$ we have to distinguish between weak, strong, and superstrong cases. The weak field can be treated by perturbation theory and described in terms of absorption of limited numbers of photons. The strong field must be treated by nonperturbative methods and is the case that interests us here. The superstrong may give rise to ionization of the molecule, which is a complication we wish to avoid in this paper.

It should be pointed out that the model contains in effect only one potential parameter $\beta$ and two field parameters $\Omega$ and $q E / \alpha \beta D$, as can be seen by transforming (1) to a dimensionless length parameter $x=\alpha R$ and a dimensionless time parameter $\tau=\omega t$. Indeed, after these substitutions we obtain, for the wave function $\phi(x, \tau)=\psi(R, t)$, the equation

$$
\begin{aligned}
i \frac{\partial \phi(x, \tau)}{\partial \tau}= & -\beta \frac{\partial^{2} \phi(x, \tau)}{\partial x^{2}}+\frac{1}{4 \beta}\left\{1-\exp \left[-\left(x-x_{0}\right)\right]\right\}^{2} \\
& \times \phi(x, \tau)-x F \cos \left(\frac{\Omega}{\omega} \tau\right) \phi(x, \tau)
\end{aligned}
$$

where

$$
F=\frac{q E \sqrt{2 D}}{\hbar \omega^{2} \sqrt{M}}
$$

We note that there are only three essential parameters in the problem: the anharmonicity of the system $\beta$ and two field parameters, the frequency parameter $\Omega / \omega$ and the amplitude parameter $F$. If the initial condition is represented by a stationary state of $H_{0}$, it depends only on the $\beta$ parameter. In particular, we see that the reduced mass $M$ is not an independent parameter; changing $M$ can be accommodated by changing $E$, i.e., different problems with the same $E / \sqrt{M}$ will yield the same results. With a given $\beta$ all energies (eigenvalues of $H_{0}$, dissociation energy, and so on) are scaled with the same factor $\hbar \omega$. More specifically, the molecular energy $\left\langle\psi\left|H_{0}\right| \psi\right\rangle$ can be written as $\hbar \omega$ times a function of the three parameters mentioned above: $\beta, F$, and $\Omega / \omega$.

The time evolution is generated by the propagator

$$
U(t)=\mathscr{T} \exp \left(-\frac{i}{\hbar} \int_{0}^{t} d s H(s)\right)
$$

where $\mathscr{T}$ is the Dyson time-ordering operator and $H$ contains the kinetic energy $T$ and the potential energy $W$, including the field-molecule interaction. The propagator is broken up into short time slices

$$
U(t)=\prod_{1}^{N} U(\varepsilon), \quad t=\varepsilon N
$$

with $N$ large and $\varepsilon$ small. This allows one to use an approximate short-time propagator for $U(\varepsilon)$ given by 


$$
\begin{aligned}
U\left(t_{j}, t_{j-1}\right) \approx & \exp \left(-\frac{i}{\hbar} T \frac{\varepsilon}{2}\right) \exp \left(-\frac{i}{\hbar} \int_{t_{j-1}}^{t_{j}} d s W(s)\right) \\
& \times \exp \left(-\frac{i}{\hbar} T \frac{\varepsilon}{2}\right)
\end{aligned}
$$

which is correct up to second order in $\varepsilon$ provided the timedependent field is well behaved (analytic) in the time variable. This is referred to as the "split operator" scheme [4]. The power of the algorithm lies in the splitting of the $W$ and $T$ terms. The action of the first is evaluated while the wave function $\psi$ is in a coordinate representation, i.e., defined by its values on a grid in coordinate space. For the second, one transforms the wave function to the momentum representation, using the discrete Fourier transform associated with the grid. The $T$ operator is multiplicative in that representation, so its action is easy to evaluate. Afterward one transforms $\psi$ back to the coordinate representation with an inverse Fourier transform. This is a viable approach because of the availability of fast Fourier transform software that performs these transformations very efficiently.

The parameters of the grid on which the $R$ dependence is represented and the time slice $\varepsilon$ are essential parameters in the computer program. Care has been taken to choose the end points of the grid and the number of grid points so as to cover sufficiently well the spatial dependence of all vibrational eigenfunctions entering the dynamics. The time slice has been determined by considering both the maximum kinetic energy involved in the motion of the wave packet and the period of the external field.

From the calculated time-dependent wave function $\psi(t)$ we can extract other quantities more amenable to physical interpretation. We have chosen to analyze the numerical results in terms of the stationary states of the free molecule and not, e.g., in terms of quasistationary Floquet states.

Our main quantity of investigation is the absorbed energy

$$
\mathscr{E}(t)=\left\langle\psi(t)\left|H_{0}\right| \psi(t)\right\rangle-\left\langle\psi(0)\left|H_{0}\right| \psi(0)\right\rangle .
$$

In this paper we shall always assume the initial state to be the ground state of the free system.

\section{RESULTS AND DISCUSSION}

In this section we shall implement the model and the computational scheme described above. We first discuss the choice of model parameters.

Let us consider a family of Morse potentials with fixed $\omega$ and variable $\beta$. This can be done by varying $D$, keeping $M$ fixed, and adapting $\alpha$ such that $\omega$ remains constant. In Table I we have listed the relevant parameters using generic values for $M\left(10^{4}\right.$ a.u. $)$ and $\omega\left(10^{-2}\right.$ a.u. $)$. The anharmonicities given here practically cover the range of realistic values [5] for common diatomic molecules, i.e., from 0.003 for $I_{2}$ to 0.027 for $\mathrm{H}_{2}{ }^{+}$.

In these diatomic molecules, the equilibrium distance $R_{0}$ varies between 1.4 a.u. for $\mathrm{H}_{2}$ and 4.46 a.u. for $\mathrm{NaCl}$. In our model we take a generic value of 2 a.u. Changing the value of $R_{0}$ does influence the problem only through an overall phase factor. The ionization potentials of diatomic molecules show [5] little variation and lie around 0.5 a.u.; therefore
TABLE I. Morse potential parameters and field amplitude parameter for cases of increasing anharmonicity and field strength with fixed $M=10^{4}$ and $\omega=10^{-2}$. All quantities are in a.u.

\begin{tabular}{lcccc}
\hline \hline Case & $D$ & $\beta$ & $E$ & $F$ \\
\hline$A 1$ & 0.5 & 0.00500 & 0.01 & 0.500 \\
$A 2$ & 0.3 & 0.00833 & 0.01 & 0.387 \\
$A 3$ & 0.1 & 0.02500 & 0.01 & 0.224 \\
& & & & \\
$B 1$ & 0.5 & 0.00500 & 0.03 & 1.500 \\
$B 2$ & 0.3 & 0.00833 & 0.03 & 1.161 \\
$B 3$ & 0.1 & 0.02500 & 0.03 & 0.672 \\
& & & & \\
$C 1$ & 0.5 & 0.00500 & 0.10 & 5.000 \\
$C 2$ & 0.3 & 0.00833 & 0.10 & 3.873 \\
$C 3$ & 0.1 & 0.02500 & 0.10 & 2.236 \\
\hline \hline
\end{tabular}

with $\mu \approx 1$ we should keep $E$ below 0.5 a.u. The intensity $I$ of the field, expressed in $\mathrm{W} / \mathrm{cm}^{2}$, is, for $E=0.01,0.03$, and 0.1 a.u., respectively, $I=3.5 \times 10^{12}, 1.05 \times 10^{13}$, and $3.5 \times 10^{14}$, which are realistic values used frequently in the literature. We shall use these three values in the subsequent numerical experiments and refer to them as cases $A, B$, and $C$ in increasing order of field amplitude. The dipole moments, on the other hand, show [6] a large variation between 0.047 a.u. for $\mathrm{CO}$ and 3.31 a.u. for $\mathrm{NaCl}$. We fix $q R_{0}$ at a generic value of 1 a.u., hence $q=0.5$ a.u. As far as the field frequency is concerned, relevant values are the multiphoton resonances, given by

$$
\Omega_{v}=\frac{E_{v}-E_{0}}{\hbar v}
$$

Using the exact Morse eigenvalues $E_{v}=\hbar \omega\left[\left(v+\frac{1}{2}\right)-\beta(v+\right.$ $\left.\frac{1}{2}\right)^{2}$, we get

$$
\Omega_{v}=\omega[1-\beta(v+1)] .
$$

During the initial time period after the interaction has been switched on, a number of photons are absorbed from the field and a number of vibrational quanta are excited in the molecule. After a later time, photons are again absorbed by the field and the molecule is deexcited. In the language of wave packets, if at time $t=0$ the molecule is in the ground state $\phi_{0}$, then at a later time $t, \psi(t)$ will represent a nonstationary wave packet consisting of a superposition of stationary states. Walker and Preston [1] already noted that the wave packet very quickly loses its coherence. Thus the dynamics of the Morse oscillator deviates from harmonic behavior already after the first few optical cycles. Nevertheless, one would expect that the photon absorption mechanism will show some regularity in the beginning before becoming, in a certain sense, stochastic due to the complicated structure of the wave function at later times. We shall not explicitly study here the wave function itself, but instead other quantities, related to the stationary states of $H_{0}$, which allow a more familiar interpretation. The efficiency of the absorption process is reflected in the value of the absorbed energy $\mathscr{E}(t)$. This quantity will show a quasiperiodic structure and we shall concentrate on the first quasiperiod. 
In order to bring out the effects specifically due to anharmonicity, it is instructive to compare our results with those of the harmonic oscillator with frequency $\omega$. The analytic solution of the driven harmonic oscillator is well known. The basic quantity here is

$$
\rho(t)=\frac{q^{2}}{2 M \hbar \omega}\left|\int_{0}^{t} E\left(t^{\prime}\right) \exp \left(i \omega t^{\prime}\right) d t^{\prime}\right|^{2},
$$

in terms of which the absorbed energy can simply be written as

$$
\mathscr{E}(t)=\hbar \omega \rho(t)
$$

In the ideal case of a monochromatic field (12), tuned exactly to the frequency of the harmonic oscillator, $\mathscr{E}(t)$ will increase without bound according to the law (neglecting terms of order $1 / \omega t$ )

$$
\mathscr{E}(t)=\frac{(q E)^{2}}{8 M} t^{2}
$$

In the case where there is a detuning $\Delta=\Omega-\omega$, we have [neglecting small, rapidly oscillating terms of order $\Delta /(\Omega$ $+\omega)$ ] a periodic behavior

$$
\mathscr{E}(t)=\frac{(q E)^{2}}{2 M \Delta^{2}} \sin ^{2}\left(\frac{\Delta t}{2}\right)
$$

In this case the maximum absorbed energy $\mathscr{E}$ and the "rising time" (we use the term coined in [7]) $T$ are related to the detuning in the obvious manner

$$
\begin{gathered}
\mathscr{E}=\frac{\left(q E_{0}\right)^{2}}{2 M \Delta^{2}}, \\
T=\frac{\pi}{\Delta}
\end{gathered}
$$

Let us first look at the typical anharmonic case $B 2$ of intermediate anharmonicity and intermediate field amplitude. In Fig. 1 we show the total molecular energy for various values of $\Omega_{v}$.

The exact curves would show a small component, rapidly oscillating with twice the field frequency. In the figure we have smoothed out these wiggles for the sake of clarity. We note the parabolalike rising of the energy, culminating in a maximum value $\mathscr{E}_{v}$ at a rising time $T_{v}$, depending on $v$. From Fig. 1 we can distinguish between three different regimes. For the low- $v$ values $(v<8)$, i.e., for high frequencies $\Omega_{v}$, the rising time and maximum energy progressively increase. For these cases there is a minimum at about $2 T_{v}$, marking a recurrence of the absorption process, that becomes less pronounced for increasing $v$. For higher- $v$ values $(v>7)$ the maximum absorbed energy decreases and very soon also the rising time decreases. Intermediate values, such as, e.g., $v=12$, do not display a regular recurrence pattern, but show a rather constant absorption and $T$ and $\mathscr{E}$ lose their significance. Finally, for large $v(v>16)$, both rising time and energy are small and the time dependence tends toward a simple Rabi oscillation.

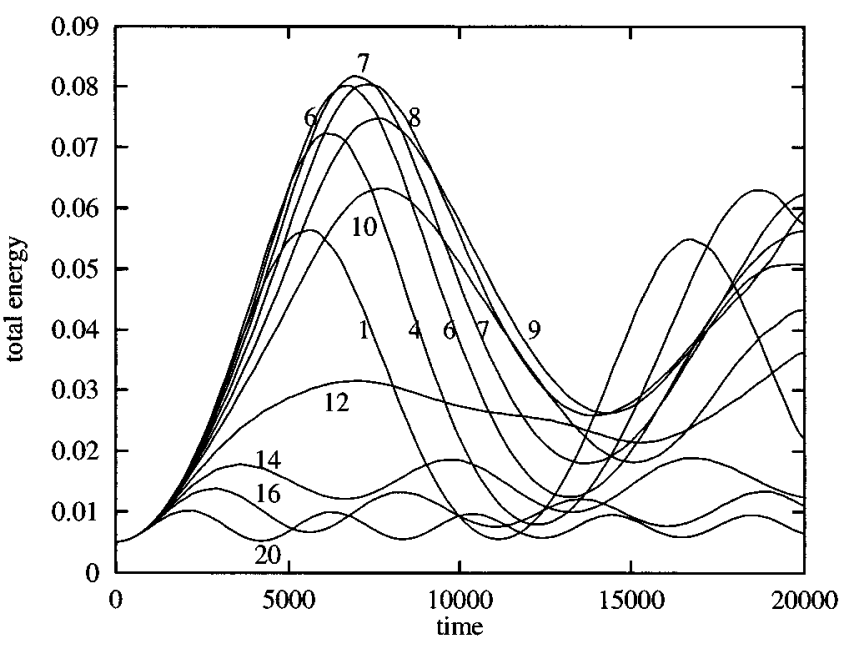

FIG. 1. Total molecular energy versus time for case $B 2$ (see Table I). The curves are labeled by the resonance order $v$, i.e., the field frequency $\Omega_{v}$ corresponds to a $v$-photon absorption. All quantities are in a.u.

In all the cases investigated, i.e., for three values of $E$ and for three values of $\beta$, mentioned in Table I, we obtain the same qualitative picture. A quantitative description can be obtained by listing, as a function of inverse anharmonicity ( $D$ parameter), the values of $v, T_{v}$, and $\mathscr{E}_{v}$ corresponding to that value of $\Omega_{v}$ which yields the greatest $\mathscr{E}_{v}$. This we show in Table II. It may be noticed that there is an approximate linear relationship between the three quantities and $D$.

The characteristics of the absorption dynamics, as exemplified in the time dependence of the molecular energy, can be analyzed in terms of an empirically defined "apparent detuning," which we determine from the rising time in analogy with formula (26)

$$
\left|\Delta_{v}\right|=\frac{\pi}{T_{v}}
$$

counting $\Delta_{v}$ negative when $T_{v}$ decreases with increasing $v$. In Figs. 2-4 we show the variation of $\Delta_{v}$ as a function of $v$ (or, equivalently, as a function of field frequency) for all the cases studied.

TABLE II. Resonance order $v$, rising time $T_{v}$, and maximum absorbed energy $\mathscr{E}_{v}$, corresponding to the optimum field frequency $\Omega_{v}$, for each of the cases listed in Table I. All quantities are in a.u.

\begin{tabular}{lcccc}
\hline \hline Case & $D$ & $v$ & $T_{v}$ & $\mathscr{E}_{v}$ \\
\hline$A 1$ & 0.5 & 5 & 17500 & 0.05 \\
$A 2$ & 0.3 & 3 & 14000 & 0.03 \\
$A 3$ & 0.1 & 2 & 11000 & 0.01 \\
$B 1$ & & & & \\
$B 2$ & 0.5 & 10 & 8500 & 0.12 \\
$B 3$ & 0.3 & 7 & 7000 & 0.08 \\
& 0.1 & 3 & 4500 & 0.03 \\
$C 1$ & & & & \\
$C 2$ & 0.5 & 25 & 3900 & 0.26 \\
$C 3$ & 0.3 & 17 & 3200 & 0.18 \\
\hline \hline
\end{tabular}




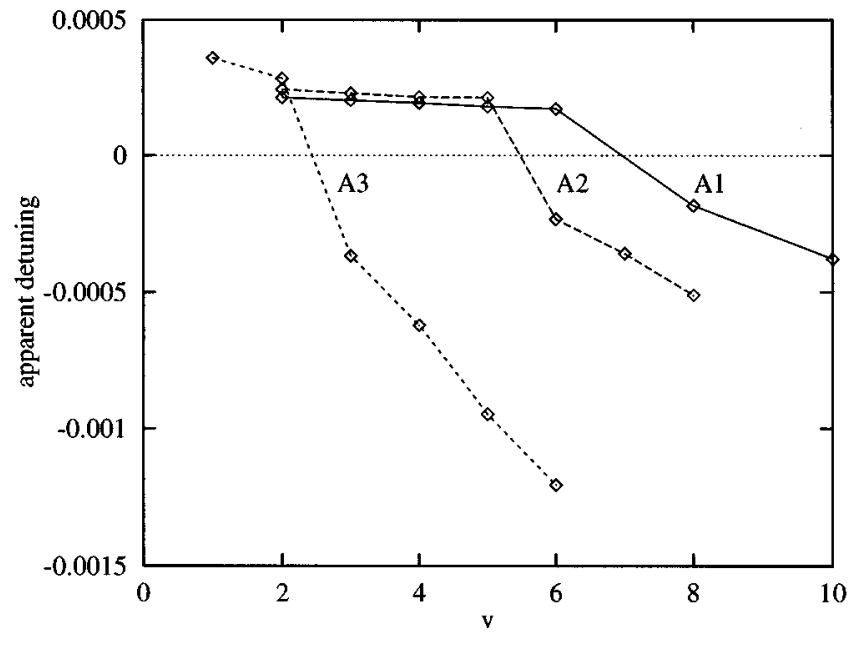

FIG. 2. Apparent detuning versus resonance order $v$ for the cases $A 1, A 2, A 3$ (see Table I) of lowest field strength $E=0.01$. All quantities are in a.u.

From these graphs we see that the distinction between the regimes described above is well defined. The same sharp transition between two frequency regions is also clear from $\mathscr{E}_{v}$ as can be observed from Fig. 5 .

The behavior of $\Delta_{v}$ can be explained by the anharmonic nature of the Morse spectrum. When, at a given field frequency, the Morse oscillator has absorbed a number $N_{v}$ of photons (on the average $N_{v}=\mathscr{E}_{v} / \hbar \Omega_{v}$ ), the wave packet exists in an excited region of the spectrum that can be simulated by a harmonic oscillator with apparent frequency $\omega_{v} \simeq \omega(1-2 \beta v)$. Because of the anharmonicity, this $\omega_{v}$ will decrease with increasing number of absorbed photons. Now, scanning the frequency $\Omega_{v}$ downward from $\Omega_{1}$, for low $v$, $N_{v}$ is larger than $v$ and hence the detuning $\Delta_{v}=\Omega_{v}-\omega_{v}$ is positive. With decreasing $\Omega_{v}, v$ approaches more and more $N_{v}$ and the detuning decreases. However, because of the unequal spacing of the vibrational levels, no $\Omega$ will yield a vanishing apparent detuning. A further decrease of $\Omega_{v}$ below

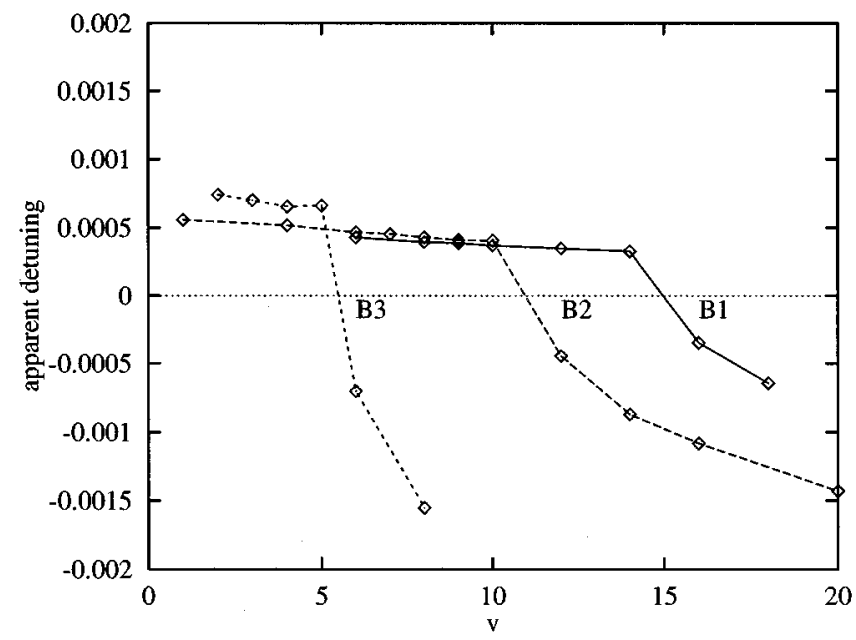

FIG. 3. Apparent detuning versus resonance order $v$ for the cases $B 1, B 2, B 3$ (see Table I) of intermediate field strength $E=0.03$. All quantities are in a.u.

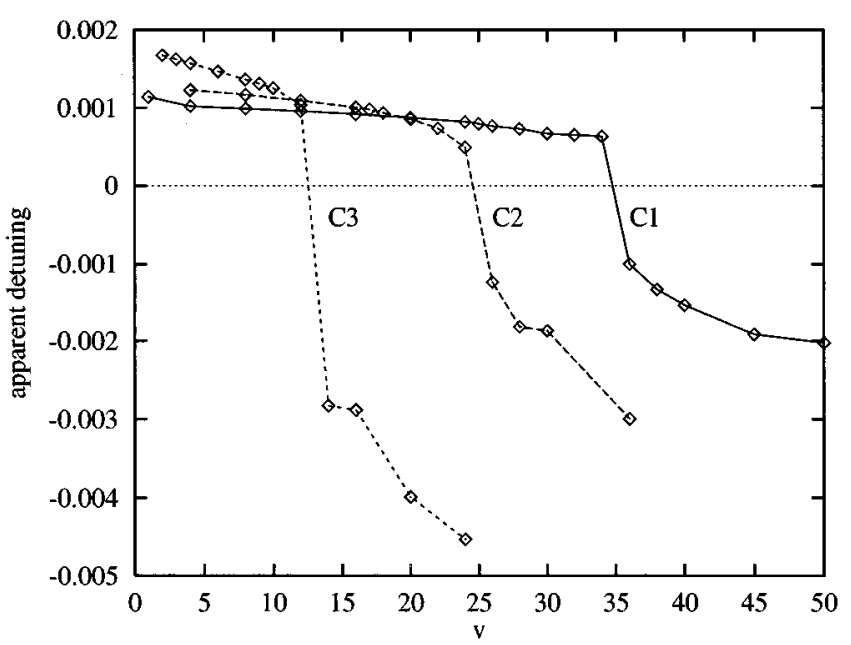

FIG. 4. Apparent detuning versus resonance order $v$ for the cases $C 1, C 2, C 3$ (see Table I) of highest field strength $E=0.10$. All quantities are in a.u.

a critical value makes the absorption of the first few photons very inefficient, so that $\omega_{v}$ rises again and makes the detuning become negative. At very high $v$ and hence very small field frequency only the first excited state is populated appreciably and the situation becomes that of a two-level system with a large detuning.

\section{CONCLUSION}

In this paper we have considered the dynamics of molecular anharmonic oscillators in dipole fields of high intensity. We have studied extensively the absorption of energy by the molecule as a function of the anharmonicity and the field strength. We have focused our attention on the rising time interval during which the molecule "climbs up" the stationary spectrum.

The characteristics of the absorption mechanism can be described in terms of an apparent detuning. This parameter is

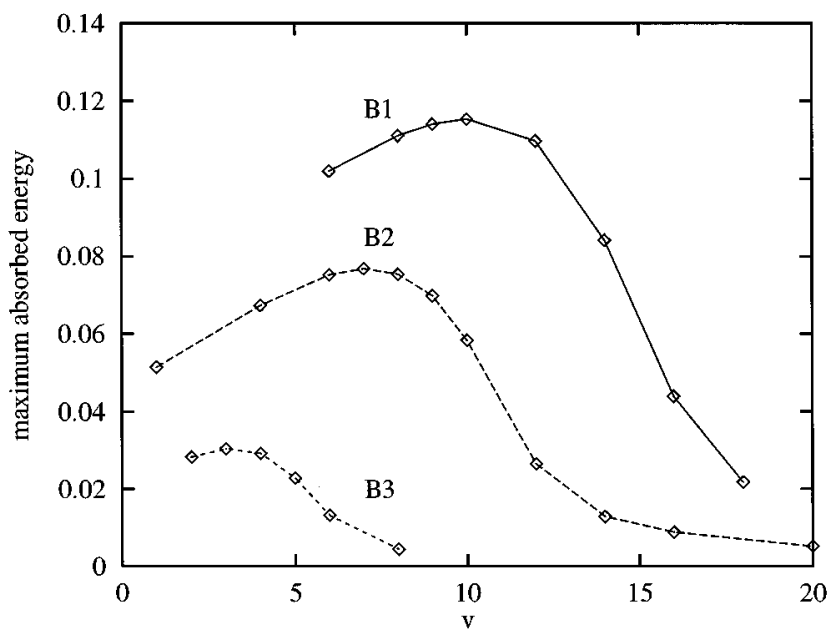

FIG. 5. Maximum absorbed energy versus resonance order $v$ for the cases $B 1, B 2, B 3$ (see Table I) of intermediate field strength. All quantities are in a.u. 


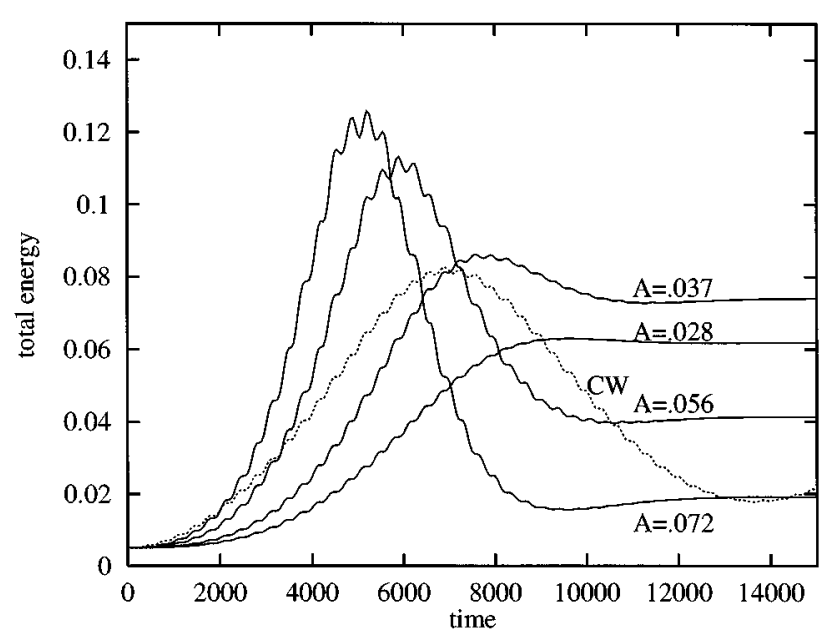

FIG. 6. Total molecular energy versus time for case $B 2$ (see Table I) under the action of a Gaussian pulse with carrier frequency $\Omega_{7}$, with pulse length 5000 and maximum amplitude at $t=5000$. The curves are labeled by the value of the amplitude $A$ (see the text). CW is the curve corresponding to the continuous wave case of Fig. 1. All quantities are in a.u.

derived empirically from the numerical data. It monitors well the different regimes that can be observed as a function of field frequency.

The analysis of the dynamics under a continuous-wave field, presented in this paper, can be used to predict the response of the anharmonic oscillator to a Gaussian pulse. Let us take an external field $E(t)$ consisting of a carrier wave of frequency $\Omega$ and an amplitude modulated by a Gaussian envelope

$$
E(t)=A \cos \Omega t \exp \left(-\frac{\left(t-t_{0}\right)^{2}}{\tau^{2}}\right)
$$

We wish to apply this pulsed field to our typical case $B 2$ and show that the parameters of (28) can be chosen a priori so as to produce a final state of the molecule with large or small absorbed energy. Let us choose the optimum resonant frequency $\Omega_{7}$. The pulse duration $\tau$ is such that the intensity $E^{2}$ has a full width at half maximum of 5000 a.u., i.e., of the same order of the rising time. We fix the pulse maximum $t_{0}=\tau$, which means that the intensity at $t=0$ is only $A^{2} / 16$, i.e., small enough to consider the field as switched on adiabatically. Then we vary the amplitude parameter A. In Fig. 6 we display the energy of the molecule versus time for various choices of $A$.

We note that by an optimum choice of $A(A=0.037)$ the molecule is left with the maximum final energy, almost equal to the maximum $\mathscr{E}_{7}$. Another choice $(A=0.072)$ results in a pulse that leaves the molecule in a state of minimum energy. A particular choice derives from the rule of equal integrated amplitude, i.e., if we take the integrated amplitude of the Gaussian pulse to be equal to that of the square pulse with duration $T$

$$
A=\frac{1}{\sqrt{\pi}} \frac{T}{\tau} E
$$

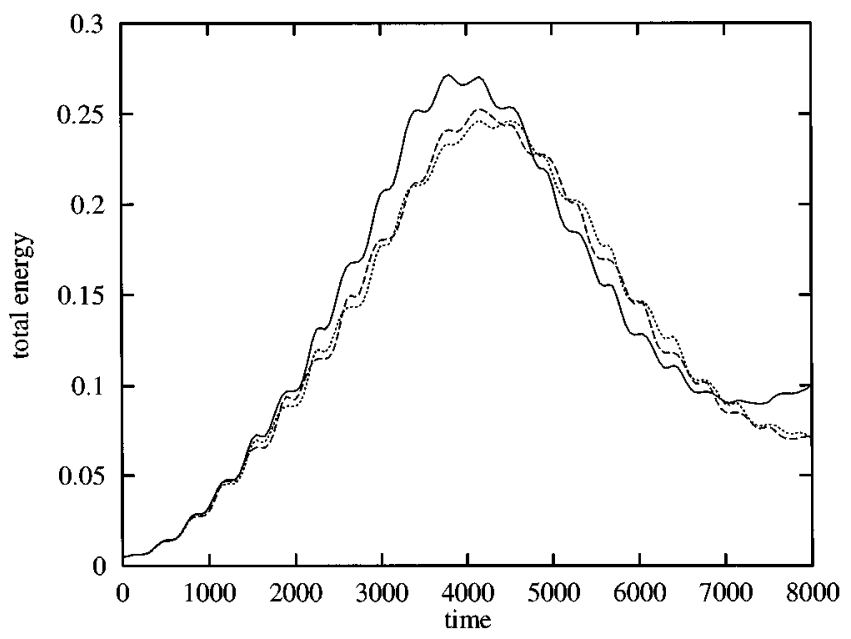

FIG. 7. Total molecular energy versus time for the case $C 1$ with $v=25$ using three different models for the dipole function: full line, linear dipole; dashed line, $\mu_{1}$ [Eq. (8)]; dotted line, $\mu_{2}$ [Eq. (9)]. All quantities are in a.u.

If now we take for $T$ the rising time, i.e., $T \simeq T_{v}$ a.u. and $A=0.028$, then it is seen that the energy absorbed during the Gaussian pulse is monotonically increasing; the interaction with the field ceases at the moment of largest absorption. The counterintuitive fact that a higher field amplitude produces less absorption is an effect of the anharmonicity. Indeed the harmonic oscillator driven by a Gaussian pulse would yield final energies that scale as $A^{2}$.

\section{ACKNOWLEDGMENTS}

We wish to thank the authors of the TEMPO computer program, J. Broeckhove and B. Feyen, for putting it at our disposal. P.V.L. gratefully acknowledges the hospitality of the Department of Chemistry, University of Pisa; his stay in Pisa has been partially supported by the Consiglio Nazionale delle Ricerche.

\section{APPENDIX}

In the foregoing study we have used the linear approximation to the dipole function. As the excited wave packet contains components from the high-energy part of the vibrational spectrum, it can extend far onto the $R$ axis and hence the wrong behavior of the linear dipole function at a large distance might pose a problem. In order to test the validity of the model we have, in Sec. II [Eqs. (8) and (9)], introduced two other models for the dipole function $\mu(R)$. In Fig. 7 we show, for all three dipole models, the total energy versus time for the case $C 1$, with $\Omega=\Omega_{25}$, where the largest effect of the tail of $\mu(R)$ is expected. It can be seen from this result, comparing the linear model with both nonlinear formulas, that the conclusions about $\mathscr{E}$ and $T$ are not affected by the choice of the dipole model. Also, comparing the nonlinear models with each other, we notice that only the absolute value of the linear term matters. The latter observation is equivalent to saying that the change of sign of the electric field (phase change of $\pi$ ) has no effect on the molecular dynamics. 
[1] R. B. Walker and R. K. Preston, J. Chem. Phys. 67, 2017 (1977); M. E. Goggin and P. W. Milonni, Phys. Rev. A 37, 796 (1988); R. Graham and M. Höhnenbach, ibid. 43, 3966 (1991); J. P. Connerade et al., J. Phys. B 25, 3771 (1992); H. P. Breuer, K. Dietz, and M. Holthaus, ibid. 24, 1343 (1991); Y. Gu and J. M. Yuan, Phys. Rev. A 36, 3788 (1987); P. A. Dando and D. Richards, J. Phys. B 23, 3179 (1990); R. Graham and M. Höhnenbach, Phys. Rev. A 45, 5078 (1991); J. Heagy and J. M. Yuan, ibid. 41, 571 (1990).

[2] The computer program TEMPO, implementing the split-operator FFT method, has been written by J. Broeckhove and B. Feyen, University of Antwerp, Departement Wiskunde-Informatica.
[3] It has been shown by G. P. Arrighini, C. Guidotti, and N. Durante, Nuovo Cimento D 9, 1219 (1987), that care should be taken in discussing occupation probabilities of vibrational states if other types of gauges are used.

[4] M. D. Feit, J. A. Fleck, and A. Steiger, J. Comput. Phys. 47, 412 (1982).

[5] K. P. Huber and G. Herzberg, Constants of Diatomic Molecules (Van Nostrand, New York, 1979).

[6] B. H. Bransden and C. J. Joachain, Physics of Atoms and Molecules (Wiley, New York, 1983).

[7] W. Jakubetz, J. Manz, and V. Mohan, Chem. Phys. 90, 3686 (1989). 